\title{
Lack of WDFY4 Aggravates Ovalbumin-Induced Asthma via Enhanced Th2 Cell Differentiation
}

\author{
Yan Li Anran Wang Feng Long Fei Gao Shang Gao Shijun Wei Ai Liu \\ Xi Li Wenjie Sun Jiangxia Li Qiji Liu
}

Key Laboratory for Experimental Teratology of the Ministry of Education, Department of Medical Genetics, School of Basic Medical Sciences, Cheeloo College of Medicine, Shandong University, Jinan, China

\section{Keywords}

WDFY4 $\cdot$ Th2 cell · Asthma

\begin{abstract}
Background: Asthma is a chronic inflammatory airway disease, and Th2 cells play an important role in asthma. WDFY4 (WDFY family member 4) is a susceptibility gene in several autoimmune diseases. Objective: In this study, the roles of WDFY4 in Th2 cell differentiation and Th2-dependent asthma were investigated. Methods: Naïve CD4 ${ }^{+} \mathrm{T}$ cells were isolated from wild-type and WDFY4-deficient mice and induced to differentiate in vitro. Subsequently, a mouse model of asthma was established by sensitization with ovalbumin. $\boldsymbol{R} \boldsymbol{e}-$ sults: Study results showed that WDFY4 deficiency could promote the differentiation of Th2 cells and the production of Th2 cytokines. WDFY4-deficient asthmatic mice showed higher levels of Th2 cytokines in the lungs and bronchoalveolar lavage fluid than wild-type mice. Moreover, infiltration of inflammatory cells, hyperplasia of goblet cells, production of mucus, and deposition of collagen were enhanced in WDFY4-deficient asthmatic mice. Conclusions: Our study demonstrates the pivotal role of WDFY4 in the pathogenesis of asthma and in Th2 cell differentiation.
\end{abstract}

(c) 2021 The Author(s)

Published by S. Karger AG, Basel

Edited by: A. Haczku, Singapore.

\section{Introduction}

Asthma is a chronic disease characterized by airway inflammation, airway hyperresponsiveness and airway remodeling [1]. It affects more than 300 million people worldwide [2]. A variety of immune cells and their corresponding cytokines are involved in the development of asthma. The important role of Th2 cells in asthma has been confirmed by previous studies [3].

Inhaled allergens can induce the differentiation of Th2 cells, which leads to the production of allergen-specific IgE by B cells, activation of mast cells, and the recruitment of eosinophils to the lungs; all these processes eventually cause persistent airway inflammation along with symptoms of asthma $[4,5]$. Th2 cells are involved in the development of asthma mainly via the secretion of Th2 cytokines, such as IL-4, IL-5 and IL-13 [6]. IL-4 is involved in the synthesis of the immunoglobulin $\operatorname{IgE}$, activation of eosinophils, production of mucus, and remodeling of the airways [7]. IL-5 is important for the differentiation, maturation and survival of eosinophils [6]. As a major regulator of asthma, IL-13 can be involved in the regulation of eosinophilic inflammation, mucus secretion and airway hyperresponsiveness [6].

Correspondence to:

Qiji Liu, liuqiji@sdu.edu.cn karger@karger.com www.karger.com/iaa

Karger $\stackrel{\text { ! }}{=}$

bOPEN ACCESS
(C) 2021 The Author(s)

Published by S. Karger AG, Basel

This is an Open Access article licensed under the Creative Commons Attribution-NonCommercial-4.0 International License (CC BY-NC) (http://www.karger.com/Services/OpenAccessLicense), applicable to the online version of the article only. Usage and distribution for commercial purposes requires written permission. 
WDFY4 (WDFY family member 4) is the fourth member of the WDFY family and is conserved among multiple species. Several studies have revealed associations between WDFY4 and autoimmune diseases, such as systemic lupus erythematosus (SLE), rheumatoid arthritis, juvenile idiopathic arthritis and clinically amyopathic dermatomyositis [8-11]. These studies imply an important role for WDFY4 in the immune system. Recently, two studies revealed the involvement of WDFY4 in cross-presentation of cell-associated antigens [12] and maintenance of B cells [13]. Global knockout mice showed that WDFY4 had no influence on the development of conventional type 1 dendritic cells (cDC1s). However, deficiency in WDFY4 could impede the cross-presentation of cell-associated antigens by $\mathrm{cDC} 1 \mathrm{~s}$ and result in an impaired antigen-specific immune response [12]. Moreover, WDFY4 could modulate B cell fate via noncanonical autophagy. Selective deficiency in WDFY4 in B cells could lead to impaired antibody responses; thus, SLE phenotypes induced by pristane were effectively alleviated in WDFY4 conditional knockout mice [13]. A new method based on two linked approaches to speculate on gene function and connect genes with biological processes was developed by Li et al. [14]. In this way, they found that WDFY4 is associated with antigen processing, $\mathrm{T}$-cell activation and the immune response in humans, rats and mice. However, this work was a mathematical study rather than a biological study. The specific effects of WDFY4 on T cells remain unclear and need to be explored in further biological studies.

Here, we demonstrated that lack of WDFY4 could enhance the differentiation of Th2 cells and the production of Th2 cytokines. As a result, WDFY4-deficient mice showed more severe airway inflammation and airway remodeling than wild-type mice. Our findings contribute to the understanding of the involvement of WDFY4 in Th2 cell differentiation and establish a link between WDFY4 and asthma.

\section{Materials and Methods}

\section{Mice}

The genetic background of the mice was C57BL/6. WDFY4deficient mice were constructed based on the LoxP-Cre system as described previously [13]. Lck-Cre ${ }^{+/-}$transgenic mice were crossed with $W d f y 4^{\text {loxp/loxp }}$ mice to generate conditional knockout (referred to as CKO) mice with selective deficiency in WDFY4 in T cells and $W d f y 4^{\text {loxp/loxp }}$ mice (referred to as WT mice) in the same litter were used for comparison. All mice were maintained under specific-pathogen-free conditions. All experiments involving mice were approved by the Animal Experiment Ethics Review Committee of Shandong University School of Medicine.
Isolation of Splenocytes and Naïve $\mathrm{CD} 4^{+} \mathrm{T}$ Cells

Spleens were processed through nylon mesh cell strainers (BD Biosciences, USA) to obtain single-cell suspension. Naïve $\mathrm{CD} 4^{+} \mathrm{T}$ cells were purified from splenocytes using the Naïve CD4 ${ }^{+} \mathrm{T}$-Cell Isolation Kit (Miltenyi Biotec, Germany) according to the manufacturer's protocol.

\section{Differentiation in vitro}

Splenocytes and naïve $\mathrm{CD} 4^{+} \mathrm{T}$ cells were cultured with platebound anti-CD3 $(10 \mu \mathrm{g} / \mathrm{mL})$ and soluble anti-CD28 $(6 \mu \mathrm{g} / \mathrm{mL})$ to differentiate the cells into Th1, Th2, and Th17 cells. For Th2 polarization, naïve $\mathrm{CD} 4^{+} \mathrm{T}$ cells were cultured with plate-bound anti-CD3, soluble anti-CD28, IL-4 (10 ng/mL), and anti-IFN- $\gamma(10$ $\mu \mathrm{g} / \mathrm{mL})$. Cells were cultured for $44 \mathrm{~h}$ and then treated with Cell Stimulation Cocktail. After $4 \mathrm{~h}$, the cells were collected for flow cytometry. Anti-CD3 and anti-CD28 were purchased from BioLegend (USA). Murine IL-4 was obtained from PeproTech (USA). Anti-IFN- $\gamma$ was obtained from BD Biosciences. Cell Stimulation Cocktail was from eBioscience (USA).

\section{Flow Cytometry}

Cultured cells were stained with anti-CD4 and then fixed with paraformaldehyde. Triton X-100 was used for permeabilization, and then the cells were stained with anti-IFN- $\gamma$, anti-IL- 4 and anti-IL-17a. Data were analyzed by using FlowJo (TreeStar, USA). Anti-CD4, anti-IFN- $\gamma$ and anti-IL-17a were purchased from BioLegend. Anti-IL-4 was purchased from BD Biosciences.

\section{Establishment of Ovalbumin-Induced Asthmatic Mice}

Mice aged 6-8 weeks were sensitized by intraperitoneal injection of $200 \mu \mathrm{g}$ ovalbumin (OVA)/ $1 \mathrm{mg} \mathrm{Al}(\mathrm{OH})_{3}$ in $200 \mu \mathrm{L}$ PBS on days 0 and 10 . From days 21 to 81 , the mice were challenged by atomization with $1 \%$ OVA for 40 min twice every week. Mice were sacrificed on day 82. OVA and $\mathrm{Al}(\mathrm{OH})_{3}$ were obtained from Sigma-Aldrich (USA) and Thermo Fisher (USA), respectively.

\section{RNA Extraction and Quantitative RT-PCR}

RNA extraction, reverse transcription and quantitative RTPCR were performed as described previously [15]. Relative expression of the target gene was normalized to the level of Gapdh, calculated by the $2^{-\Delta \Delta \mathrm{Ct}}$ method. The sequences of the primers $\left(5^{\prime}-3^{\prime}\right)$ were as follows: Gata3 TCCCGTCCTACTACGGAAAC/CCGCCAGAGAAGAGGATGAA; $c$-Maf GGAGACCGACCGCATCATC/TCATCCAGTAGTAGTCTTCCAGG; IL-4 GTTGTCATCCTGCTCTTCTTTCTC/CTGTGGTGTTCTTCGTTGCT; IL-5 GACAAGCAATGAGACGATGAG/AATAGCATTTCCACAGTACCC; IL-13 TGAGCAACATCACACAAGACC/GGCCTTGCGGTTACAGAGG; and Colla1 AGGGCGAGTGCTGTGCTTT/CCCTCGACTCCTACATCTTCTGA.

Western Blot Analysis

Western blotting was performed to detect the expression of GATA3 in Th2 cells. The details were described previously [15]. Antibodies against mouse GATA3 and GAPDH were purchased from Proteintech (USA).

\section{ELISA}

The concentration of IL-4 in the medium supernatant and bronchoalveolar lavage fluid (BALF) was detected by using the Mouse IL-4 Precoated ELISA Kit (DAKEWE, China) according to the manufacturer's protocol. 


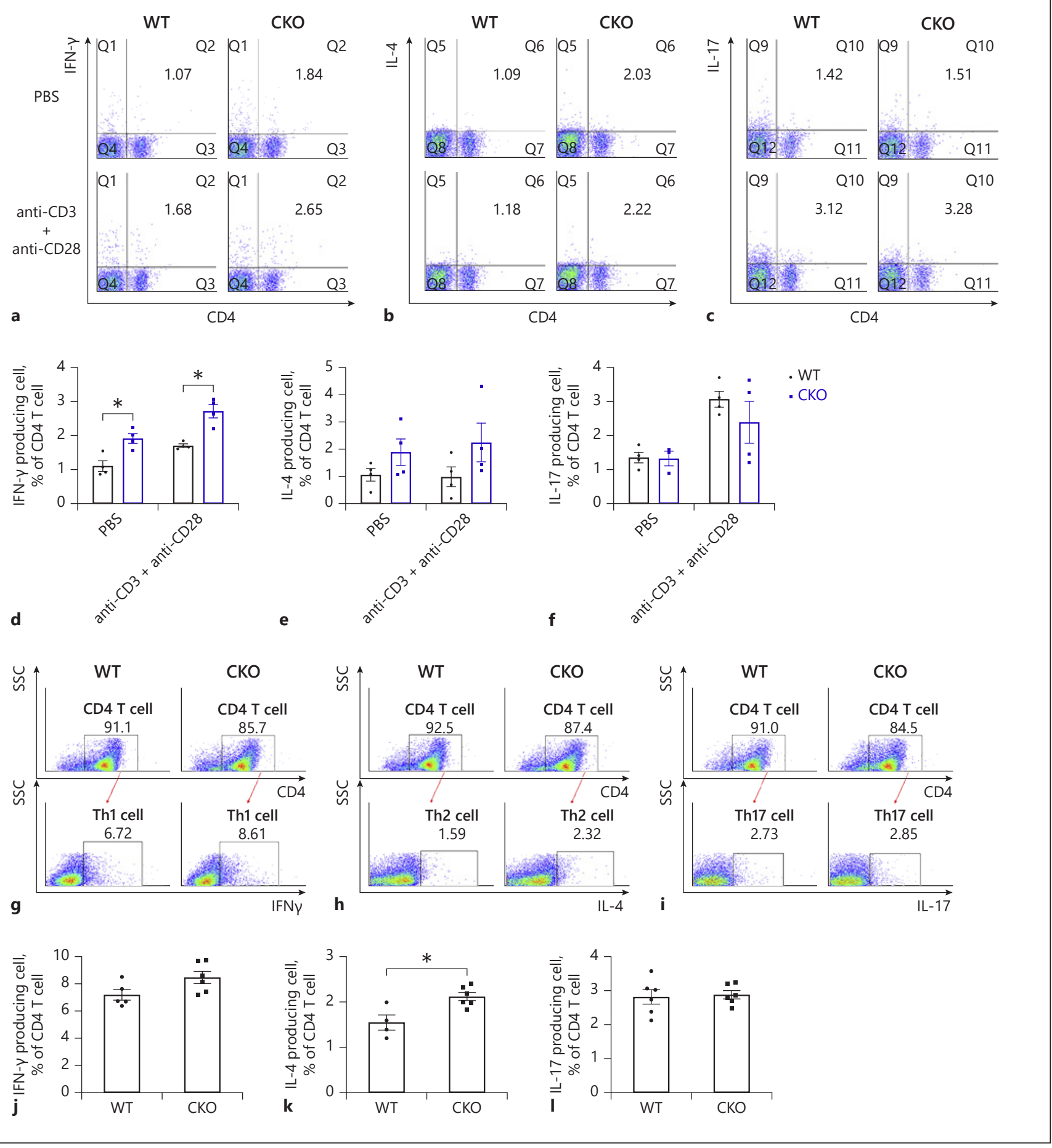

Fig. 1. a-c Splenocytes from WT mice and CKO mice were treated with anti-CD3 $(10 \mu \mathrm{g} / \mathrm{mL})$ and anti-CD28 $(6 \mu \mathrm{g} / \mathrm{mL})$ for $48 \mathrm{~h}$. Intracellular cytokine staining was performed to assess the proportions of Th cells by flow cytometry. The numbers in the FACS pseudocolour images indicate the percentages of Th cells in the $\mathrm{CD} 4^{+} \mathrm{T}$-cell population. d-f Statistical results for a-c. Data are shown as the mean $\pm \operatorname{SEM}(n=4: 4) .{ }^{*} p<0.05$, Mann-Whitney $\mathrm{U}$ test. g-i Naïve $C D 4^{+} \mathrm{T}$ cells were purified and treated with anti-
CD3 $(10 \mu \mathrm{g} / \mathrm{mL})$ and anti-CD28 $(6 \mu \mathrm{g} / \mathrm{mL})$ for $48 \mathrm{~h}$. Intracellular cytokine staining was performed to assess the proportions of Th cells by flow cytometry. In the FACS pseudocolour images, the numbers adjacent to the outlined areas indicate the percentages of CD4 ${ }^{+} \mathrm{T}$ cells or Th cells. $\mathbf{j}-\mathbf{I}$ Statistical results for $\mathbf{g - i}$. Data are shown as the mean $\pm \operatorname{SEM}(n=2: 2)$. Every mouse was evaluated in $2-3$ replicates. ${ }^{*} p<0.05$, Mann-Whitney U test. Only statistically significant results are marked. 
Fig. 2. Naïve $\mathrm{CD}^{+} \mathrm{T}$ cells were cultured with plate-bound anti-CD3 $(10 \mu \mathrm{g} / \mathrm{mL})$, soluble anti-CD28 (6 $\mu \mathrm{g} / \mathrm{mL}), \mathrm{IL}-4$ (10 ng/ $\mathrm{mL})$ and anti-IFN- $\gamma(10 \mu \mathrm{g} / \mathrm{mL})$. After 48 $\mathrm{h}$, the cells were collected for studies. a Representative mRNA levels of Gata3 and $c-M a f$ were confirmed by quantitative RT-PCR. Student's $t$ test. b Western blotting was performed to detect the level of GATA 3 in cultured Th 2 cells. c The relative expression of IL-4, IL-5 and IL-13 was examined by quantitative RT-PCR. ${ }^{*} p<0.05$, Student's $t$ test. d The medium supernatant of cultured Th2 cells was collected and the concentration of IL-4 was measured by ELISA. Mann-Whitney U test. All data are shown as the mean \pm SEM $(n=2: 2)$. For quantitative RT-PCR, every mouse was evaluated in 4 replicates, and for ELISA, every mouse was evaluated in 2 replicates. Only statistically significant results are marked.

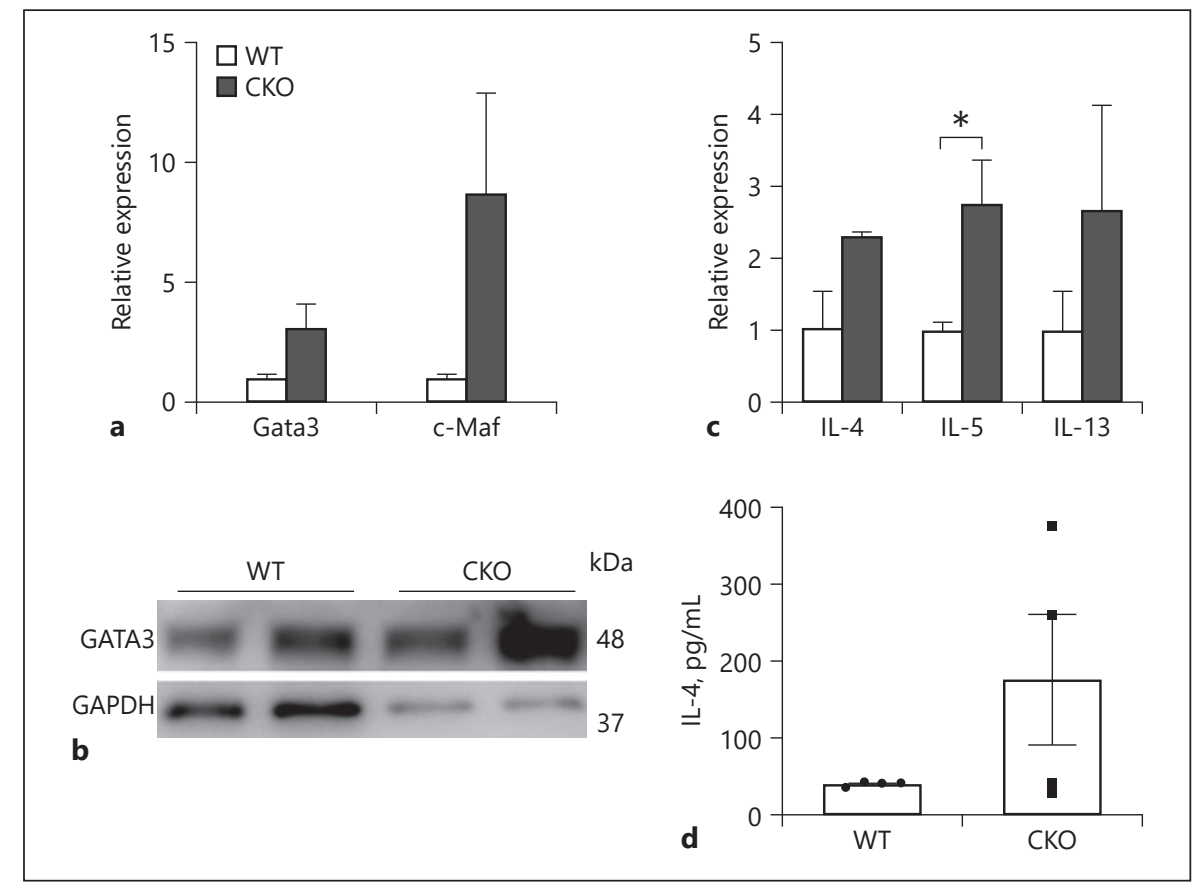

Histopathological Analysis

The lungs of asthmatic mice were fixed with paraformaldehyde and dehydrated with an alcohol series. Subsequently, the samples were embedded in paraffin and cut into $5-\mu \mathrm{m}$ sections. Then, the sections were stained with hematoxylin and eosin (HE), periodic acid-Schiff or Sirius red. Images were captured with a microscope (Olympus, Japan).

\section{Statistical Analysis}

All data were recorded as the mean \pm SEM. GraphPad Prism (GraphPad Software, USA) was used to generate graphs. Data were analyzed by using Student's $t$ test or the Mann-Whitney U test, and $p<0.05$ was considered statistically significant. All experiments were repeated independently at least three times.

\section{Results}

\section{Lack of WDFY4 Promotes Th2 Cell Differentiation}

To explore the influences of WDFY4 on T cells, WDFY4-deficient mice were constructed based on the LoxP-Cre system as described previously [13]. Mice with selective deficiency in WDFY4 in T cells were constructed by crossing $L c k-C r e^{+/-}$mice and $W d f y 4^{\text {loxp/loxp }}$ mice. Conditional knockout mice were referred to as CKO mice, and $W d f y 4^{\text {loxp/loxp }}$ mice (referred to as WT mice) were used as controls.

Splenocytes from WT mice and CKO mice were treated with plate-bound anti-CD3 and soluble anti-CD28 for activation and cultured in vitro for $48 \mathrm{~h}$. Then flow cy- tometry was performed to detect the proportions of Th cells. As shown in Figure 1, cells treated with anti-CD3 and anti-CD28 showed higher percentages of Th cells than cells treated with PBS. After activation, more Th1 cells and Th2 cells and fewer Th17 cells were observed in WDFY4-deficient T cells (Fig. 1a-f).

To further confirm the above results, naïve $\mathrm{CD} 4^{+} \mathrm{T}$ cells were isolated from WT mice and CKO mice using magnetic beads and activated in vitro. Consistently, deficiency in WDFY4 promoted the differentiation of naïve $\mathrm{CD}^{+}{ }^{\mathrm{T}} \mathrm{T}$ cells into Th2 cells (Fig. 1h, k). However, there were no significant differences in the proportions of Th1 cells or Th17 cells between WT T cells and WDFY4-deficient T cells (Fig. 1g, i, j, l). Overall, lack of WDFY4 promotes the differentiation of Th2 cells.

\section{WDFY4-Deficient Th2 Cells Express Relatively High}

\section{Levels of Th2 Cytokines}

Naïve $\mathrm{CD}^{+} \mathrm{T}$ cells from WT mice and CKO mice were purified and cultured under Th2 polarization conditions to clarify the involvement of WDFY4 in Th2 cell differentiation. Quantitative RT-PCR results showed upregulated expression of Gata3 and c-Maf, important transcription factors for Th2 cell differentiation, in WDFY4deficient Th2 cells compared with WT Th2 cells (Fig. 2a). GATA3 is defined as the specific transcription factor for Th2 cell differentiation. Western blotting was performed to detect the expression of GATA3, and higher levels of 
Fig. 3. a Experimental protocol for the induction of an OVA-induced asthmatic mouse model. b The mRNA levels of Gata3 and $c$-Maf in the lungs of asthmatic mice were detected by quantitative RT-PCR. ${ }^{*} p<0.05$, Student's $t$ test. c The BALF of asthmatic mice was collected, and the concentration of IL- 4 was measured by ELISA. ${ }^{*} p<0.05$, Mann-Whitney U test. All data are shown as the mean $\pm \operatorname{SEM}(n=3: 4)$. For quantitative RT-PCR, every mouse was evaluated in 4 replicates. Only statistically significant results are marked. OVA, ovalbumin; BALF, bronchoalveolar lavage fluid.

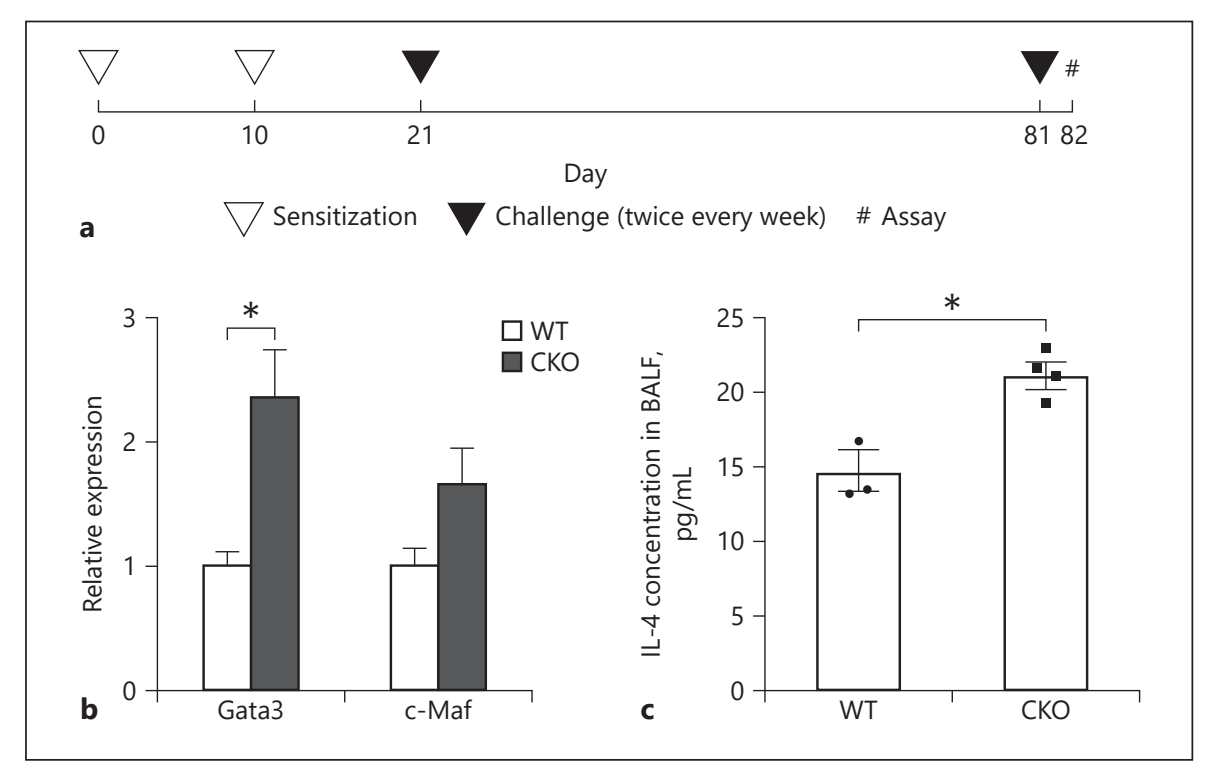

GATA3 were observed in WDFY4-deficient Th2 cells (Fig. 2b).

Th2 cells participate in immune responses mainly by secreting cytokines, such as IL-4, IL-5 and IL-13. We found that deficiency in WDFY4 could promote the production of Th2 cytokines (Fig. 2c). The medium supernatant of cultured Th2 cells was collected, and the concentration of IL- 4 in the medium supernatant was measured by ELISA. The result showed higher level of IL-4 in the medium supernatant of WDFY4-deficient Th2 cells than in that of WT Th2 cells (Fig. 2d). The above data indicate the important roles of WDFY4 in the differentiation of Th2 cells and production of Th2 cytokines.

Deficiency in WDFY4 Promotes the Production of Th2 Cytokines in Asthmatic Mice

To determine whether WDFY4 is involved in asthma by influencing the differentiation of Th2 cells, an OVAinduced asthmatic mouse model was established (Fig. 3a). After challenge, the mice treated with OVA showed head and face itching, fidgeting, and crouching. The expression of Gata3 and $c$-Maf in the lungs was detected by quantitative RT-PCR, and upregulated expression of Gata3 and c-Maf was observed in WDFY4-deficient asthmatic mice (Fig. 3b). Moreover, the BALF was collected, and the level of IL-4 was higher in the BALF of WDFY4deficient asthmatic mice than in that of WT asthmatic mice (Fig. 3c). These data reveal that deficiency in WDFY4 promotes the production of Th2 cytokines in asthmatic mice.

WDFY4 Participates in Th2 Cell

Differentiation and Asthma
Aggravated Airway Inflammation Was Observed in WDFY4-Deficient Asthmatic Mice

Airway inflammation is the pathological basis of bronchial asthma [16]. Hematoxylin and eosin (HE) staining was performed to evaluate airway inflammation in asthmatic mice. As shown in Figure 4a, infiltration of inflammatory cells was observed in asthmatic mice, and inflammatory cell infiltration was increased in WDFY4-deficient asthmatic mice compared with WT asthmatic mice (Fig. 4a). Goblet cell hyperplasia and increased mucus production are the characteristic features of allergic inflammation [6]. Mucus overproduction and goblet cell hyperplasia were observed in asthmatic mice, and WDFY4-deficient asthmatic mice exhibited more severe phenotypes (Fig. 4b). These results suggest that deficiency in WDFY4 can aggravate airway inflammation in asthmatic mice.

\section{Deficiency in WDFY4 Promotes Airway Remodeling in Asthmatic Mice}

Airway remodeling is one of the common features of chronic asthma, and collagen deposition is an important feature of airway remodeling. Sirius red staining was performed, and we found that the collagen deposition in WDFY4-deficient asthmatic mice was more serious than that in WT asthmatic mice (Fig. 4c). Consistently, quantitative RT-PCR result showed upregulated expression of Colla1 in WDFY4-deficient asthmatic mice (Fig. 4d). In general, deficiency in WDFY4 promotes airway remodeling in asthmatic mice.

Int Arch Allergy Immunol 2021;182:1089-1096 DOI: $10.1159 / 000516970$ 


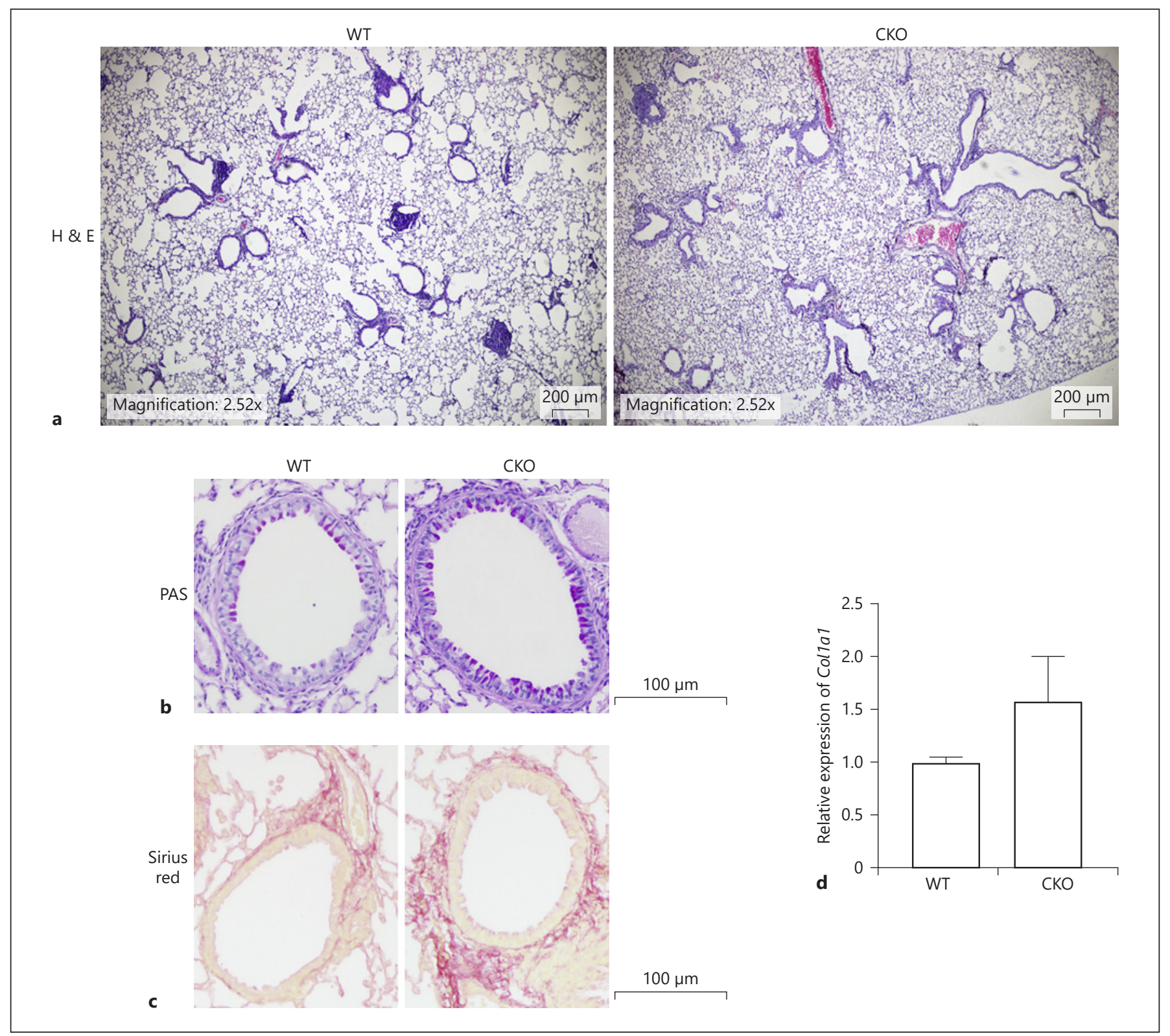

Fig. 4. a Lung sections were stained with HE; magnification, $40 \times$. Bar $=200 \mu \mathrm{m}$. b Lung sections were stained with PAS; magnification, $100 \times$. Bar $=100 \mu \mathrm{m}$. c Lung sections were stained with Sirius red; magnification, 100×. Bar $=100 \mu \mathrm{m}$. d The mRNA level of Colla1 in the lungs of asthmatic mice was detected by quantitative RT-PCR. Data are shown as the mean \pm SEM. 4-8 mice were sacrificed for histopathological analysis, and 4 mice were sacrificed for quantitative RT-PCR. For quantitative RT-PCR, every mouse was evaluated in 4 replicates. HE, hematoxylin and eosin; PAS, periodic acid-Schiff.

\section{Discussion}

The first report on WDFY4 was a genome-wide association study that identified variants in WDFY4 associated with SLE in Asian populations [8]. Subsequently, the association between WDFY4 and SLE has been confirmed in several populations [17-19]. Zhao et al. [20] revealed the mechanism by which WDFY4 leads to SLE susceptibility. They found that the functional variant rs877819 of WDFY4 could specifically bind to the transcription factor YY1 and that the affinity between rare gene A and YY1 was significantly lower than 
that of allele $\mathrm{G}$, thus downregulating the transcriptional activity of rare gene A. In addition to SLE, other autoimmune diseases, such as rheumatoid arthritis, juvenile idiopathic arthritis and clinically amyopathic dermatomyositis, have been reported to be associated with WDFY4 [9-11]. As a susceptibility gene in various autoimmune diseases, WDFY4 must be important for immune cells and immune responses. Although Li et al. [14] found that WDFY4 is associated with T-cell activation and the immune response in humans, rats and mice, the detailed effects of WDFY 4 on T cells remain unclear. Th2 cells are involved in humoral immunity, allergic reactions and chronic inflammation. In this paper, we generated CKO mice with selective deficiency in WDFY4 in T cells. We found that compared to WT cells, WDFY4-deficient T cells were more likely to differentiate into Th2 cells after activation or under Th2 polarization conditions.

Transcription factors play an important role in regulating the differentiation of Th cells. GATA3 is defined as the main regulator of Th2 cell differentiation and is upregulated during Th2 cell differentiation [21]. Knockout or inhibition of GATA3 can not only suppress the differentiation of Th2 cells but also inhibit the production of Th2 cytokines and airway hyperresponsiveness [22-24]. c-Maf, another transcription factor involved in Th2 cell differentiation, is a potential target of GATA3 [25]. We found that WDFY4-deficient Th2 cells expressed higher levels of Gata3 and c-Maf than WT Th2 cells. It has been reported that GATA3 and c-Maf can promote the production of IL-4, IL-5 and IL-13 [24, 26, 27]. Consistently, higher levels of Th2 cytokines were observed in WDFY4-deficient Th2 cells and WDFY4-deficient asthmatic mice than in the corresponding WT controls.

Previous studies have shown that Th2 cells play an important role in asthma. Inhaled allergens can induce the differentiation of Th2 cells, which leads to the production of allergen-specific IgE by B cells, activation of mast cells, and recruitment of eosinophils to the lungs; all these processes eventually cause persistent airway inflammation along with symptoms of asthma $[4,5]$. Airway inflammation is the pathological basis of asthma [16]. Inflammatory cell infiltration, goblet cell hyperplasia and increased mucus production are the characteristic features of airway inflammation in asthma. Histopathological analysis showed that the amounts of infiltrating inflammatory cells and goblet cells in WDFY4-deficient asthmatic mice were greater than those in WT asthmatic mice.

WDFY4 Participates in Th2 Cell

Differentiation and Asthma
Unlike in most studies, a mouse model of chronic asthma, not acute asthma, was established in this study. In addition to airway inflammation, airway remodeling was observed in the mouse model of chronic asthma. Airway remodeling is defined as changes in the constituents, structure and amount of airway wall cells and extracellular components [28] and is induced by repeated injury/repair processes [29]. Collagen deposition is one of the characteristic features of airway remodeling. Prolonged and repeated allergen stimulation can lead to collagen deposition, which can result in structural alterations. As a consequence, airway contraction is restricted and airway hyperresponsiveness occurs [30]. Consistently, we observed more severe collagen deposition in WDFY4-deficient asthmatic mice than in WT asthmatic mice.

In this study, we demonstrate that lack of WDFY4 can promote the differentiation of Th2 cells and the production of Th2 cytokines, thus aggravating OVA-induced asthma. Our results establish a link between WDFY4 and asthma and provide a novel idea for the study of disease pathogenesis and treatment.

\section{Statement of Ethics}

The animal experiments conformed to internationally accepted standards and were approved by the Animal Experiment Ethics Review Committee of Shandong University School of Medicine (reference number: 201402030).

\section{Conflict of Interest Statement}

The authors have no conflicts of interest to declare.

\section{Funding Sources}

This work was supported by the National Natural Science Foundation of China (81873878, 32070586, 81021001, 81300835, and 81671114) and Shandong Natural Science Foundation (2018CXGC1211 and 2015GSF118050).

\section{Author Contributions}

Conception and design: Y. Li, X. Li, W. Sun, J. Li, and Q. Liu. Development of methodology: J. Li, X. Li, W. Sun, and Q. Liu. Acquisition of data: Y. Li, A. Wang, S. Gao, S. Wei, and A. Liu. Analysis and interpretation of data: Y. Li, A. Wang, F. Long, and F. Gao. Writing, review and/or revision of the manuscript: Y. Li and Q. Liu. 


\section{References}

1 Debeuf N, Haspeslagh E, van Helden M, Hammad H, Lambrecht BN. Mouse models of asthma. Curr Protoc Mouse Biol. 2016 Jun 1;6(2):169-84.

2 Croisant S. Epidemiology of asthma: prevalence and burden of disease. Adv Exp Med Biol. 2014;795:17-29.

3 Bosnjak B, Stelzmueller B, Erb KJ, Epstein MM. Treatment of allergic asthma: modulation of Th2 cells and their responses. Respir Res. 2011 Aug 25;12:114.

4 Holgate ST. Pathogenesis of asthma. Clin Exp Allergy. 2008 Jun;38(6):872-97.

5 Lambrecht BN, Hammad H. The immunology of asthma. Nat Immunol. 2015 Jan;16(1): $45-56$.

6 Zhang Q, Wang L, Chen B, Zhuo Q, Bao C, Lin L. Propofol inhibits NF-kappaB activation to ameliorate airway inflammation in ovalbumin (OVA)-induced allergic asthma mice. Int Immunopharmacol. 2017 Oct;51: 158-64.

7 Bagnasco D, Ferrando M, Varricchi G, Passalacqua G, Canonica GW. A critical evaluation of Anti-IL-13 and Anti-IL-4 strategies in severe asthma. Int Arch Allergy Immunol. 2016;170(2):122-31.

8 Yang W, Shen N, Ye DQ, Liu Q, Zhang Y, Qian XX, et al. Genome-wide association study in Asian populations identifies variants in ETS1 and WDFY4 associated with systemic lupus erythematosus. PLoS Genet. $2010 \mathrm{Feb}$ 12;6(2):e1000841.

9 Zhang Y, Bo L, Zhang H, Zhuang C, Liu R. E26 transformation-specific-1 (ETS1) and WDFY family member 4 (WDFY4) polymorphisms in Chinese patients with rheumatoid arthritis. Int J Mol Sci. 2014 Feb 17;15(2): 2712-21.

10 McIntosh LA, Marion MC, Sudman M, Comeau ME, Becker ML, Bohnsack JF, et al. Genome-wide association meta-analysis reveals novel juvenile idiopathic arthritis susceptibility loci. Arthritis Rheumatol. 2017 Nov; 69(11):2222-32.
11 Kochi Y, Kamatani Y, Kondo Y, Suzuki A, Kawakami E, Hiwa R, et al. Splicing variant of WDFY4 augments MDA5 signalling and the risk of clinically amyopathic dermatomyositis. Ann Rheum Dis. 2018 Apr;77(4):602-11.

12 Theisen DJ, Davidson JT, Briseño CG, Gargaro M, Lauron EJ, Wang Q, et al. WDFY4 is required for cross-presentation in response to viral and tumor antigens. Science. 2018 Nov 9;362(6415):694-9.

13 Yuan Q, Li Y, Li J, Bian X, Long F, Duan R, et al. WDFY4 is involved in symptoms of systemic lupus erythematosus by modulating $B$ cell fate via noncanonical autophagy. J Immunol. 2018 Nov 1;201(9):2570-8.

14 Li H, Rukina D, David FPA, Li TY, Oh CM, Gao AW, et al. Identifying gene function and module connections by the integration of multispecies expression compendia. Genome Res. 2019 Dec;29(12):2034-45.

15 Xin Q, Li J, Dang J, Bian X, Shan S, Yuan J, et al. miR-155 deficiency ameliorates autoimmune inflammation of systemic lupus erythematosus by targeting S1pr1 in Faslpr/lpr mice. J Immunol. 2015 Jun 1;194(11):543745.

16 Filosso PL, Turello D, Magnoni MS. [Chronic inflammation and airway remodeling in asthma. Importance of an early treatment]. Minerva Pediatr. 2003 Aug;55(4):323-9.

17 Wang C, Ahlford A, Järvinen TM, Nordmark G, Eloranta ML, Gunnarsson I, et al. Genes identified in Asian SLE GWASs are also associated with SLE in Caucasian populations. Eur J Hum Genet. 2013 Sep;21(9):994-9.

18 Lessard CJ, Sajuthi S, Zhao J, Kim K, Ice JA, $\mathrm{Li} \mathrm{H}$, et al. Identification of a systemic lupus erythematosus risk locus spanning ATG16L2, FCHSD2, and P2RY2 in Koreans. Arthritis Rheumatol. 2016 May;68(5):1197-209.

19 Odhams CA, Cortini A, Chen L, Roberts AL, Viñuela A, Buil A, et al. Mapping eQTLs with RNA-seq reveals novel susceptibility genes, non-coding RNAs and alternative-splicing events in systemic lupus erythematosus. Hum Mol Genet. 2017 Mar 1;26(5):1003-17.
20 Zhao H, Yang W, Qiu R, Li J, Xin Q, Wang X, et al. An intronic variant associated with systemic lupus erythematosus changes the binding affinity of Yinyang1 to downregulate WDFY4. Genes Immun. 2012 Oct;13(7):53642.

21 Zhu J. Transcriptional regulation of Th2 cell differentiation. Immunol Cell Biol. 2010 Mar-Apr;88(3):244-9.

22 Zhang DH, Yang L, Cohn L, Parkyn L, Homer R, Ray P, et al. Inhibition of allergic inflammation in a murine model of asthma by expression of a dominant-negative mutant of GATA-3. Immunity. 1999 Oct;11(4):473-82.

23 Pai SY, Truitt ML, Ho IC. GATA-3 deficiency abrogates the development and maintenance of T helper type 2 cells. Proc Natl Acad Sci U S A. 2004 Feb 17;101(7):1993-8.

24 Zhu J, Min B, Hu-Li J, Watson CJ, Grinberg A, Wang $\mathrm{Q}$, et al. Conditional deletion of Gata3 shows its essential function in $\mathrm{T}(\mathrm{H}) 1$ $\mathrm{T}(\mathrm{H}) 2$ responses. Nat Immunol. 2004 Nov; 5(11):1157-65.

25 Hwang ES, White IA, Ho IC. An IL-4-independent and CD25-mediated function of cmaf in promoting the production of Th2 $\mathrm{cy}$ tokines. Proc Natl Acad Sci U S A. 2002 Oct 1; 99(20):13026-30

26 Kim JI, Ho IC, Grusby MJ, Glimcher LH. The transcription factor c-Maf controls the production of interleukin- 4 but not other Th2 cytokines. Immunity. 1999 Jun;10(6):745-51.

27 Li B, Tournier C, Davis RJ, Flavell RA. Regulation of IL-4 expression by the transcription factor JunB during $T$ helper cell differentiation. EMBO J. 1999 Jan 15;18(2):420-32.

28 Warner SM, Knight DA. Airway modeling and remodeling in the pathogenesis of asthma. Curr Opin Allergy Clin Immunol. 2008 Feb;8(1):44-8.

29 Hirota N, Martin JG. Mechanisms of airway remodeling. Chest. 2013 Sep;144(3):1026-32.

30 Royce SG, Cheng V, Samuel CS, Tang ML. The regulation of fibrosis in airway remodeling in asthma. Mol Cell Endocrinol. 2012 Apr $4 ; 351(2): 167-75$. 